\title{
Anesthesiologist-Administered Gastrointestinal Sedation: Time to Put It to Sleep?
}

\author{
Kilian Friedrich $^{1} \cdot$ Wolfgang Stremmel ${ }^{1}$
}

Received: 30 April 2015/ Accepted: 6 May 2015/Published online: 19 May 2015

(C) Springer Science+Business Media New York 2015

In the ongoing debate regarding sedation administered during gastrointestinal (GI) endoscopy, supporters of sedation administered by anesthesia providers (AAP) argue that anesthesiologists best handle complications involving the airways. Furthermore, the product label for propofol, the most commonly used sedative for GI endoscopy, states: "it should be administered only by persons trained in the administration of general anesthesia." Supporters of nonanesthesiologist-administered propofol (NAAP) sedation, specifically nurse-administered propofol administration under the guidance of the endoscopist, argue that the safety profile of NAAP is equivalent to AAP while being costeffective. For low- and average-risk procedures, overwhelming worldwide data support the hypothesis that NAAP has a comparable safety profile to AAP if sedation is administered after proper training, and according to implemented guidelines [1].

In this issue of Digestive Diseases and Sciences, Goudra et al. [2] conducted a comparative meta-analysis evaluating respiratory complications of AAP- or NAAP-administered sedation during advanced GI endoscopic procedures. The authors provide data with regard to 3018 advanced GI endoscopic procedures carried out using NAAP-administered sedation and 2374 AAP-administered procedures. Regarding non-advanced GI endoscopy, a prior populationbased analysis studying complications of sedation reported that the use of anesthesiologist services was associated

Kilian Friedrich

Kilian.Friedrich@med.uni-heidelberg.de

1 Department of Gastroenterology, University Hospital of Heidelberg, Im Neuenheimer Feld 420, 69120 Heidelberg, Germany with a higher complication frequency, specifically respiratory adverse events [3], a finding not supported by the data reported by Goudra et al. who described similar pooled hypoxia rates in patients undergoing advanced endoscopic procedures sedated with propofol, irrespective of administering provider, although the rate of airway interventions such as jaw thrust, chin lift, mask ventilation, or endotracheal intubation was higher in the AAP group. As AAPs administered higher doses of propofol, patient satisfaction and endoscopist satisfaction were increased if AAP-administered sedation was used. Satisfaction might have been further influenced by the observed preference for co-administration of adjuvants used in AAP sedation, whereas propofol alone was preferentially administered in the NAAP group. Nevertheless, higher dosages of propofol administered during GI sedation usually increase complication rates [4], particularly hypotension and bradycardia, which were not evaluated in the study by Goudra et al. It would have been interesting to see whether the observed beneficial satisfaction of AAP sedation came at the expense of higher cardiovascular adverse events.

Limitations to the study of Goudra et al. include the exclusive selection of non-randomized prospective trials for analysis as there exist no randomized control trials comparing hypoxia, airway intervention rates, and patient satisfaction after endoscopic sedation. Furthermore, potential biases such as differences in baseline patient and endoscopist characteristics were not analyzed.

While NAAP sedation for GI endoscopy has successfully and safely been introduced to countries such as Germany [5], it remains unlikely that NAAP will be soon introduced in other countries despite literature supporting its safety and lower cost, due to financial and political issues. When moderate sedation is provided by a US endoscopist, a separate charge for this service is not permitted 
under Medicare or Medicaid, whereas the commercial anesthesia fee for colonoscopy is $\sim 60 \%$ higher than the endoscopist's fee [6]. Therefore, many endoscopists are not willing to shoulder the added responsibilities and insurance fees attributable to sedation administration and monitoring without commensurate compensation.

Meta-analysis of sedation regimens is usually limited by heterogeneity resulting from varying sedation regimen, the use of additional anesthetics such as morphine derivatives or benzodiazepines, and potential bias in that more complex patients are more likely to be referred to an anesthesiologist [2]. Although this heterogeneity is also present in the study by Goudra et al., its results complement and expand the existing data supporting the general use of AAP-administered sedation in GI endoscopy.

\section{References}

1. Rex DK, Deenadayalu VP, Eid E, et al. Endoscopist-directed administration of propofol: a worldwide safety experience. Gastroenterology. 2009;137:1229-1237.
2. Goudra BG, Singh PM, Gouda G, et al. Safety of non-anesthesia provider-administered propofol (NAAP) sedation in advanced gastrointestinal endoscopic procedures: comparative meta-analysis of pooled results. Dig Dis Sci. (Epub ahead of print). doi:10.1007/ s10620-015-3608-x.

3. Cooper GS, Kou TD, Rex DK. Complications following colonoscopy with anesthesia assistance: a population-based analysis. JAMA Intern Med. 2013;173:551-556.

4. Sieg A, Beck S, Scholl SG, et al. Safety analysis of endoscopistdirected propofol sedation: a prospective, national multicenter study of 24,441 patients in German outpatient practices. $J$ Gastroenterol Hepatol. 2014;29:517-523.

5. Behrens A, Kainzinger FA, Nölling T, et al. S3 Guideline on "sedation in gastrointestinal endoscopy": how much does the new guideline cost in everyday hospital work? A calculation model and analysis of implementation in 2011 among ALGK members. Z Gastroenterol. 2012;50:1002-1007.

6. Hannenberg AA. Payment for procedural sedation. Curr Opin Anaesthesiol. 2004;17:171-176. 